\title{
Clinical Profile of Patients with Exaggerated Blood Pressure Response During Treadmill Stress Test and Its Correlation with Risk Factors for Coronary Artery Disease
}

\author{
Angelica Dela Cruz, Dioscoro Bayani, Arnolfo Tomas, Elmer Jasper Llanes \\ Section of Cardiology, Department of Medicine, Philippine General Hospital, University of the Philippines, Manila, Philippines \\ Email address: \\ jellydelacruz1897@yahoo.com (A. D. Cruz), ding_imed2006@yahoo.com (D. Bayani), thenameisarn@gmail.com (A. Tomas), \\ ejb11011@yahoo.com (E. J. Llanes)
}

\section{To cite this article:}

Angelica Dela Cruz, Dioscoro Bayani, Arnolfo Tomas, Elmer Jasper Llanes. Clinical Profile of Patients with Exaggerated Blood Pressure Response During Treadmill Stress Test and Its Correlation with Risk Factors for Coronary Artery Disease. Cardiology and Cardiovascular Research. Vol. 4, No. 3, 2020, pp. 80-84. doi: 10.11648/j.ccr.20200403.11

Received: May 10, 2020; Accepted: May 28, 2020; Published: June 8, 2020

\begin{abstract}
This study determined the prevalence and clinical profile of patients with Exaggerated Blood Pressure Response (EBPR) during Treadmill Exercise Test (TET) and its correlation with traditional risk factors of Coronary Artery Disease (CAD). Methodology: This is a cross-sectional study performed in Out Patient Department of Philippine General Hospital of patients 19 years old and above. Clinical profile was obtained and TET parameters including blood pressure at rest, during exercise and recovery period were determined. Results: There were 226 patients included in the study. The mean age is 52 and $121(53.5 \%)$ were females. There were $108(47.8 \%)$ with hypertension, $55(24.3 \%)$ were smoker, $73(32.3 \%)$ with dyslipidemia, $1(0.4)$ with hyperuricemia, $31(13.7)$ with diabetes, $51(22.6 \%)$ with family history of CAD and $2(3.9 \%)$ with history of supraventricular tachycardia (SVT). The prevalence of EBPR is $20.21 \%$. Those with EBPR are usually female 31 $(81.6 \%)$ and likely hypertensive $22(57.9 \%)$. Female sex and abnormal BMI were the significant predictors of EBPR with pvalue of 0.001 and 0.042 respectively. Conclusion: The prevalence of ESBR is $20.21 \%$. The EBPR is strongly correlated with female sex and abnormal BMI. Further screening of these patient and aggressive management by adapting new HTN guidelines might help to reduce cardiovascular risk.
\end{abstract}

Keywords: Treadmill Exercise Test, Exaggerated Blood Pressure Response, Coronary Artery Disease

\section{Introduction}

Exercise electrocardiogram testing is among the most fundamental and widely used test for the evaluation of patients with cardiovascular disease [1]. It is routinely done in outpatient department and the initial procedure of choice in patients with normal resting electrocardiogram who are capable of adequate exercise to diagnose cardiovascular disease [2]. The vast majority of treadmill Exercise Test (TET) is performed in OPD patients with symptoms of chest pain and if ischemic heart disease is suspected. Review of census of patients who underwent treadmill exercise test in PGH for the past years ranges to 20-30 per month and most of them were referral from OPD clinic.

EBPR during exercise has been associated with an increased incidence of hypertension and CAD [3]. It was concluded that disproportionate blood pressure response to exercise is the strongest predictor of left ventricular hypertrophy [4]. The mechanism is not wholly understood but believed to be closely related to underlying systemic endothelial dysfunction, reduced proximal aortic compliance, and high exercise-induced Angiotensin II level [5]. The aforementioned mechanisms were also evident in traditional risk factors of CAD (old age, hypertension, diabetes, dyslipidemia, smoking and family history of CAD), hence EBPR may be indicative of future development of hypertension and adverse cardiac events.

EBPR is defined by the following: Systolic BP greater than $210 \mathrm{mmHG}$ in men and greater than $190 \mathrm{mmHg}$ in women $^{1}$; Exaggerated exercise systolic BP (Ex-SBP, stage 2): Sex-specific, age-predicted systolic BP $\geq 95$ th percentile during the second stage of exercise ${ }^{2}$; Exaggerated exercise 
diastolic BP (Ex-DBP, stage 2): Sex-specific, age-predicted diastolic $\mathrm{BP} \geq 95$ th percentile during the second stage of exercise $^{2}$; Elevated recovery systolic BP (Rec-SBP, 3 minutes): Sex-specific, age-predicted systolic $B P \geq 95$ th percentile at the third minute of the recovery phase ${ }^{2}$; Elevated recovery diastolic BP (Rec-DBP, 3 minutes) Sexspecific, age-predicted diastolic $\mathrm{BP} \geq 95$ th percentile at the third minute of the recovery phase [2].

Most of the data used to evaluate blood pressure as a risk factor for future cardiovascular morbidity and mortality are based on blood pressure recorded during at rest. Studies showed that blood pressure during exercise is much accurate and less influenced by nervousness and external factors such as noise, talking and when the circulatory system is challenged by a large increase in cardiac output and peripheral blood flow, hence, hypertension could be unveiled and distinguished from a temporary blood pressure increase during rest [6].

Associations between the blood pressure response to exercise, endothelial function, aortic stiffness, and neurohormonal response in healthy individuals was studied and showed that higher levels of angiotensin II at peak exercise were observed in individuals with EBPR. These findings suggest that angiotensin II is implicated in the pathophysiology of blood pressure hyper-responsiveness to submaximal exercise. On the other hand, another study showed that increase in angiotensin II level during exercise was found significantly higher in patients with EBPR, but no significant difference in other clinical variables such as BMI, smoking, diabetes mellitus and dyslipidemia [7]. Additionally, higher aortic stiffness was observed in individuals with EBPR compared with individuals with normal blood pressure response [8].

Comparison of the risk factors for hypertension between normotensive subjects with EBPR and those who exhibit a normal curve of blood pressure during TET was done. Age and BMI were the risk factors that independently influenced EBPR during TET [9].

Since exaggerated SBP response to exercise may foresee the risk of hypertension and cardiovascular risk, identification of patients with EBPR during TET and correlating it with the traditional risk factors of CAD may offer strong prediction and further identification of patients at risk for cardiovascular disease.

This study will enable to maximize the use of TET in admitted and OPD patients that needs further evaluation. Results of this study will promote primary prevention and decrease the incidence and detrimental sequelae of potential cardiovascular events. Likewise, aggressive lifestyle modification, Blood pressure monitoring at home and close follow up may be advised.

Angiotensin II is implicated in the pathogenesis of EBPR, according to the Trial of Preventing Hypertension (TROPHY), Angiotensin Receptor Blocker therapy significantly delayed incident of hypertension. Diagnosis of hypertension in Asian population using ACC/AHA 2017 Guidelines and aggressive medical management may be considered in this group of people provided no contraindication.
The primary objective of this study is to describe the clinical profile of patients with EBPR in TET according to gender, age, BMI, presence of traditional risk factors for coronary heart disease: hypertension, diabetes, dyslipidemia, history of smoking and family history of coronary heart disease. It also aimed to determine the prevalence of exaggerated systolic pressure response among patients undergoing Treadmill Stress Test and its correlation with the traditional risk factors of CAD including hypertension, Diabetes, dyslipidemia, history of smoking and family history of coronary heart disease.

\section{Materials and Methods}

This is a prospective cross sectional study of patients data bases in University of the Philippines-Philippine General Hospital (UP-PGH) from February 2019 to August 2019. The UP-PGH is tertiary hospital capable of performing Treadmill Exercise Test. Included in this study were patients from OPD who were 19 years old and above who were seen and referred by their attending physicians and underwent TET as diagnostic test to elicit abnormalities not present at rest and to determine the likelihood of CAD as well as monitoring of chronotropic competence. Excluded are those patients with clinical evidence of $\mathrm{CAD}$, heart failure, valvular or congenital heart disease as these diseases were already established. Convenience sampling was used in this study.

The protocol was approved by the University of the Philippines Manila Research Ethics Board (UPMREB) Panel. There were no direct benefits to the patients and management were not affected in this study. Written Informed consent was provided, explained and obtained by the cardiology fellow and nurse in charge who were directly involved in the test and management of patients. Included patients were assessed and sent by their physician. Appropriateness of the test was identified and written (based of AUC) on the TET request form. No monetary compensation were given to the patients included in this study. The researcher and nurse in charge were the only personnel who can access the data and information of the patients. Data were stored in the filing cabinet with lock inside the cardiovascular section unit. There were no identified ethical issues in this study since it only involves the chart review of qualified patients.

\section{Results}

Table 1. Clinical Profile of Patients who underwent Treadmill Stress Test.

\begin{tabular}{ll}
\hline & Frequency $(\mathbf{n}=\mathbf{2 2 6})$ \\
\hline Age (in years) & \\
$<55$ & $115(51 \%)$ \\
$\geq 55$ & $111(49 \%)$ \\
Mean $\pm \mathrm{SD}=51.89 \pm 11.96$ & \\
Sex & \\
Male & $105(46.5 \%)$ \\
Female & $121(53.5)$ \\
Hypertension & $108(47.8 \%)$ \\
Diabetes Mellitus & $31(13.7 \%)$ \\
Dyslipidemia & $73(32.3 \%)$ \\
\hline
\end{tabular}




\begin{tabular}{ll}
\hline & Frequency (n=226) \\
\hline Smoker & $55(24.3 \%)$ \\
Family History of CAD & $51(22.6 \%)$ \\
Others & \\
Asthma & $3(1.3 \%)$ \\
BPH & $1(0.4 \%)$ \\
Breast CA & $1(0.4 \%)$ \\
COPD & $1(0.4 \%)$ \\
Gout & $1(0.4 \%)$ \\
Hypothyroidism & $2(0.9 \%)$ \\
PSVT & $2(0.9 \%)$ \\
RHD & $1(0.4 \%)$ \\
SVT & $1(0.4 \%)$ \\
Thyroidectomy & $1(0.4 \%)$ \\
BMI & \\
Underweight & $3(1.3 \%)$ \\
Normal & $62(27.4 \%)$ \\
Overweight & $67(29.6 \%)$ \\
Obese I & $62(27.4 \%)$ \\
Obese II & $32(14.2 \%)$ \\
\hline
\end{tabular}

Table 1 shows the clinical profile of patients who underwent treadmill stress test. A total of 226 subjects were included in the study. The age ranged from 19 to 74 years with a mean age of 52 years old.

Table 2. Clinical Profile of Patients with Exaggerated Systolic Blood Pressure.

\begin{tabular}{|c|c|c|c|}
\hline & \multicolumn{2}{|l|}{ EBPR } & \multirow{2}{*}{ p-value* } \\
\hline & $(+)(n=38)$ & $(-)(n=188)$ & \\
\hline \multicolumn{4}{|l|}{ Age (in years) } \\
\hline$<55$ & $19(50.0 \%)$ & $96(51.1 \%)$ & \multirow[t]{2}{*}{$0.90(\mathrm{NS})^{\dagger}$} \\
\hline$\geq 55$ & $19(50.0 \%)$ & $92(48.9 \%)$ & \\
\hline \multicolumn{4}{|l|}{ Sex } \\
\hline Male & $7(18.4 \%)$ & $98(52.1 \%)$ & \multirow[t]{2}{*}{$<0.001(\mathrm{~S})^{\dagger}$} \\
\hline Female & $31(81.6 \%)$ & $90(47.9 \%)$ & \\
\hline Hypertension & $22(57.9 \%)$ & $86(45.7 \%)$ & $0.17(\mathrm{NS})^{\dagger}$ \\
\hline Diabetes Mellitus & $6(15.8 \%)$ & $25(13.3 \%)$ & $0.68(\mathrm{NS})^{\dagger}$ \\
\hline Dyslipidemia & $10(26.3 \%)$ & $63(33.5 \%)$ & $0.39(\mathrm{NS})^{\dagger}$ \\
\hline Smoker & $5(13.2 \%)$ & $50(26.6 \%)$ & $0.08(\mathrm{NS})^{\dagger}$ \\
\hline Family History of CAD & $9(23.7 \%)$ & $42(22.3 \%)$ & $0.86(\mathrm{NS})^{\dagger}$ \\
\hline $\mathrm{HF} /$ Prev ACS & 0 & $13(6.9 \%)$ & $0.13(\mathrm{NS})^{\ddagger}$ \\
\hline CVD & 0 & $7(3.7 \%)$ & $0.60(\mathrm{NS})^{\ddagger}$ \\
\hline Hyperuricemia & $1(2.6 \%)$ & $4(2.1 \%)$ & $1.00(\mathrm{NS})^{\ddagger}$ \\
\hline CKD & $1(2.6 \%)$ & 0 & $0.16(\mathrm{NS})^{\ddagger}$ \\
\hline \multicolumn{4}{|l|}{ Others } \\
\hline Asthma & $2(5.2 \%)$ & $1(2.6 \%)$ & $0.07(\mathrm{NS})^{\ddagger}$ \\
\hline $\mathrm{BPH}$ & 0 & $1(2.6 \%)$ & $1.00(\mathrm{NS})^{\ddagger}$ \\
\hline Breast CA & 0 & $1(2.6 \%)$ & $1.00(\mathrm{NS})^{\ddagger}$ \\
\hline COPD & $1(2.6 \%)$ & 0 & $0.16(\mathrm{NS})^{\ddagger}$ \\
\hline Gout & 0 & $1(2.6 \%)$ & $1.00(\mathrm{NS})^{\ddagger}$ \\
\hline Hypothyroidism & $1(2.6 \%)$ & $1(2.6 \%)$ & $0.31(\mathrm{NS})^{\ddagger}$ \\
\hline PSVT & $22(5.2 \%)$ & 0 & $0.02(\mathrm{~S})^{+}$ \\
\hline RHD & 0 & $1(2.6 \%)$ & $1.00(\mathrm{NS})^{\ddagger}$ \\
\hline PSVT & $1(2.6 \%)$ & 0 & $0.16(\mathrm{NS})^{\ddagger}$ \\
\hline Thyroidectomy & 0 & $1(2.6 \%)$ & $1.00(\mathrm{NS})^{\ddagger}$ \\
\hline \multicolumn{4}{|l|}{ BMI } \\
\hline Underweight & 0 & $3(1.6 \%)$ & \multirow{5}{*}{$0.78(\mathrm{NS})^{\dagger}$} \\
\hline Normal & $8(21.1 \%)$ & $54(28.7 \%)$ & \\
\hline Overweight & $13(34.2 \%)$ & $54(28.7 \%)$ & \\
\hline Obese I & $11(28.9 \%)$ & $51(27.1 \%)$ & \\
\hline Obese II & $6(15.8 \%)$ & $26(13.8 \%)$ & \\
\hline
\end{tabular}

$* \mathrm{p}>0.05$ - Not significant; $\mathrm{p} \leq 0.05$-Significant.

Data presented as Mean $\pm \mathrm{SD}$, (medians) were computed as needed; or as frequency $(\%)$.

${ }^{\dagger}$ Chi-square test; ${ }^{*}$ Fisher Exact test.
Table 2 shows the prevalence of EBPR 38 (20.21\%). Half of patients with EBPR were under 55 years old 19 (50\%) and mostly were females $31(81.6 \%)$. Patients with hypertension $22(57.9 \%)$ and those with dyslipidemia 10 (26.3\%) were significant in that higher proportion of subjects with EBPR. PSVT is correlated with EBPR but multivariate analysis showed insignificant result (Table 3).

Table 3. Multivariate Analysis of the Different Variables as Predictor of Exaggerated Systolic Blood Pressure Response among Patients Undergoing Treadmill Stress Test.

\begin{tabular}{llll}
\hline & OR & 95\% CI & p-value* \\
\hline Age & 1.00 & $0.97-1.04$ & $0.88(\mathrm{NS})$ \\
BMI (Abnormal) & 1.39 & $1.01-1.79$ & $0.042(\mathrm{~S})$ \\
Gender (Female) & 6.29 & $2.13-18.54$ & $0.001(\mathrm{~S})$ \\
HTN & 1.91 & $0.81-4.46$ & $0.14(\mathrm{NS})$ \\
Smoker & 1.13 & $0.36-3.56$ & $0.84(\mathrm{NS})$ \\
Other Com: PSVT & $2.492 \mathrm{E} 10$ & 0 & $0.99(\mathrm{NS})$ \\
HF/Prev ACS & 0 & 0 & $0.99(\mathrm{NS})$ \\
CKD & $4.617 \mathrm{E} 9$ & 0 & $0.99(\mathrm{NS})$ \\
Constant & 0.003 & --- & $0.001(\mathrm{~S})$ \\
\hline
\end{tabular}

Logistic Regression.

Table 3 shows the multivariate analysis of the different variables as predictor of exaggerated systolic blood pressure response among patients undergoing treadmill stress test. The results showed that only abnormal BMI and female gender were significant predictors of EBPR as shown by the $p$ values 0.042 and 0.001 respectively. The risk of patients with abnormal BMI for EBPR was 1.39x higher than those with normal BMI $(\mathrm{OR}=1.39$; 95\% CI: 1.01- 1.79; $\mathrm{p}=0.042)$. The risk for females for EBPR was at least $6 \mathrm{x}$ higher than males $(\mathrm{OR}=6.29 ; 95 \%$ CI: $2.13-18.54 ; \mathrm{p}=0.001)$. From the above result, a model can be generated predicting EBPR by significant variables BMI (abnormal) and gender (female) given by: Model $\rightarrow$ EBPR=BMI (abnormal) + Gender (female).

\section{Discussion}

Despite new guidelines and treatment approach, hypertension is still considered as public health problem in the Philippines together with cardiovascular disease. It is recommended to base the diagnosis of hypertension on repeated office BP, out of the office BP measurement or with ambulatory BP measurement (ABPM) based on 2018 ESC/ESH Hypertension Guidelines. At present, threshold for diagnosis and treatment of HTN still vary in practicing physician due to discrepancies of new hypertension guidelines.

Blood pressure measurement in the office setting were usually recorded after the patient rested for few minutes but sometimes forgotten to consider other circumstances that might alter BP response. Hence, ABPM is better and can give estimate of the true, mean blood-pressure and blood-pressure variability in the setting of different activities.

On the other hand, measuring BP during TET may be of help in determining the risk of development of hypertension 
and cardiovascular disease particularly on those patients exhibiting EBPR. Based on the common consensus, blood pressure recorded during exercise is less influenced by nervousness and external factors such as noise, talking and when circulatory system is challenge by large increase in cardiac output and peripheral blood flow [10].

Studies claimed that EBPR to treadmill exercise was independently associated with risk of future hypertension [11], but some published papers proved that there was no correlation between the two. On the other hand, age, hypercholesterolemia and increased BMI have positive correlation with EBPR [12]. The discrepancies of results could be due to differences in the definition of EBPR in different reports.

In our study, multivariate analysis showed that female sex and BMI are strongly correlated with EBPR. This result could be explained by the fact that majority of women recently are not engaging with physical activity. According to Kumamyika et al. [10], there is decline in the number of women who exercise and more than $60 \%$ of women in the United States are less active and obese [13]. This report regarding female gender and obesity may be similar to model we have generated in the results predicting EBPR by significant variables (abnormal BMI and female gender). Based on Framingham study, an increased BMI has direct relation to increased risk of about $75 \%$ of men and $65 \%$ of women with HTN as a direct result of overweight [12]. The INTERSALT Study showed an association with BMI and both Blood pressure regardless of age, alcohol consumption, smoking, and urinary sodium excretion [14].

Hypertension, diabetes, smoking history, family history of $\mathrm{CAD}$ and dyslipidemia did not predict the development of future hypertension.

Two patients with PSVT both happened to have EBPR, but multivariate analysis showed that SVT did not predict EBPR. Theoretically, EBPR could be due to increased sympathetic nervous system and renin-angiotensin-aldosterone system (RAAS) action during exercise, mediating heart rate and BP augmentations [15]. Harmful effects of hypertensive response to exercise on structure and function of left ventricle has been stated in numerous studies. It is due to continuous exposure to increase pressure loads to left ventricle, which may result in global subendocardial ischemia due to disparity between demand and supply from excessive rate-pressure stress [16].

At present, there is controversy in diagnosis of hypertension and when to start medical treatment in Asian population due to recent released ACC/AHA 2017 hypertension guidelines. Lower cut offs and applicability issues were the main points but no question on lifestyle and diet modification in these group of patients. Starting antihypertensive medication in patients exhibiting EBPR and who met the 2017 ACC/AHA hypertensive guidelines criteria may be considered due the pathophysiologic mechanism of EBPR in exercise such as endothelial dysfunction and aortic stiffness which were also seen in patients with traditional risk factors of coronary heart disease particularly old age, hypertension, diabetes, dyslipidemia, smoker and those with family history of CAD.

\section{Conclusion}

The prevalence of ESBR is 20.21\%. Female sex and abnormal BMI are strongly with corelated with EBPR. Multivariate analysis showed that none of the traditional risk factors of CAD significantly predicts EBPR. A study looking at cardiovascular outcomes of patients with EBPR should also be done. A primary prevention plan is still suggested in patients with EBPR without co-morbidities to prevent the progression from a well state to the disease. Lifestyle and diet modification are strongly advised, and individualized medical treatment may be considered in this population provided no contraindication.

\section{References}

[1] Braunwalds Heart Disease A textbook of Cardiovascular Medicine $10^{\text {th }}$ edition.

[2] Jagmeet Et Al, Blood Pressure Response During Treadmill Testing as a Risk Factor for New-Onset Hypertension, The Framingham Heart Study.

[3] Mehmet Akif Duzenli et al: Blunted heart rate recovery is associated with exaggerated blood pressure response during exercise testing.

[4] Kokkinos P, Pittaras A, narayan P, et al. Exercise capacity and blood pressure associations with left ventricular mass in prehypertensive individuals. Hypertension. 2007; 49: 55-61.

[5] Nicolaos T. et al. Exaggerated Exercise Blood Pressure Response and Future Cardiovascular Disease, 2013.

[6] MH Criqui, WL Haskell, G Heiss, HA Tryoler, P Green, CJ Rubenstein: Predictors of systolic blood pressure response to treadmill exercise: The Lipid Research Clinics Program Prevalence Study.

[7] MichealDoumas et al. Exaggerated Blood Pressure Response to Exercise: Will It Ever Be Ready for Prime Time? The Journal of Clinical Hypertension. August 2015.

[8] Chi Young et Al, Exagerrated Blood Pressure Respinse to Exercise is Associated with Augmented Rise of Angitensin II During Exercise. Journal of American College of Cardiology 2008.

[9] De Lima SG at al: Exaggerated blood pressure response during exercise treadmill testing: functional and hemodynamic features, and risk factors.

[10] Kumanyika S. Special issues regarding obesity in minority populations. Ann Intern Med. 1994; 119: 6550-6554. [PubMed].

[11] O'Donnell CJ, Ridker PM, Glynn RJ, Berger K, Ajani U, Manson JE, Hennekens CH. Hypertension and borderline isolated systolic hypertension increase risks of cardiovascular disease and mortality in male physicians. Circulation. 1997; 95: 1132-1137.

[12] Sae Young Jae et al; During Treadmill Testing as a Predictor of Future Hypertension in Men: A Longitudinal Study; American Journal of Hypertension, Volume 28, Issue 11, 1 November 2015. 
[13] Matthews CE, Pate RR, Jackson KL, Ward DS, Macera CA, Kohl HW, et al. Exaggerated blood pressure response to dynamic exercise and risk of future hypertension. J Clin Epidemiol. 1998; 51: 29-35. doi: 10.1016/S08954356(97)00223-0. [PubMed] [CrossRef].

[14] Lund-Johansen P. Hemodynamics in early essential hypertension. Acta Med Scand 1967; 181 (suppl 482): 1-101.
[15] Miyai N, Arita M, Miyashita K, Morioka I, Shiraishi T, Nishio I. Blood pressure response to heart rate during exercise test and risk of future hypertension. Hypertension. 2002; 39: 761766. doi: 10.1161/hy0302.105777. [PubMed] [CrossRef].

[16] De Lima SG at al: Exaggerated Blood Pressure Response to Dynamic Exercise and Risk of Future Hypertension. 